\title{
Assessing the Potential Role of Calcitonin in Cancer
}

\author{
'DB Nandini, ${ }^{2}$ Roopa S Rao, ${ }^{3}$ Shankargouda B Patil, ${ }^{4}$ A Thirumal Raj
}

How to cite this article: Nandini DB, Rao RS, Patil SB, Raj AT. Assessing the Potential Role of Calcitonin in Cancer. World J Dent 2018;9(4):253-254.

\section{Source of support: Nil}

\section{Conflict of interest: None}

Parafollicular cells of the thyroid gland produce calcitonin (CT) which is responsible for regulating the calcium metabolism. Apart from its physiological functions, recent studies have shown that $\mathrm{CT}$ plays a major role in carcinogenesis. ${ }^{1-4}$ The role of CT in carcinogenesis varies significantly according to the type of cancer. ${ }^{3}$ The present text summarizes the varying effects of CT in cancer.

Calcitonin and calcitonin receptors (CTRs) play an important role in regulating tumor development, cell proliferation, adhesion, migration, metastasis, cell survival, and apoptosis. The CTRs belong to G mitogen-activated protein-coupled seven transmembrane domain receptor (GPCR) superfamily. The CTRs control the activity of protein kinases (MAPK) superfamily which gets activated by exposure to mutagens. These include extracellular signal-regulated kinases 1 (Erk1) and 2 (Erk2) (or p44 MAPK and p42 MAPK), c-Jun NH (2)-terminal kinases, p38 MAPK, and Erk5 (or BMK). Among these kinases, Erk1 and Erk2 are the ones which regulate cellular responses like cell proliferation. The Erks are activated by a phosphorylation cascade brought about by initiation of ras, a proto-oncogene exposed to a mutagen. The GPCR may signal ras via various signaling pathways that may vary in different cell types and lines. ${ }^{4}$

${ }^{1}$ Department of Oral Pathology \& Microbiology, Dental College Regional Institute of Medical Sciences, Imphal, Manipur, India

${ }^{2}$ Department of Oral Pathology \& Microbiology, Faculty of Dental Sciences, Ramaiah University of Applied Sciences, Bengaluru Karnataka, India

${ }^{3}$ Division of Oral Pathology, Department of Maxillofacial Surgery and Diagnostic Sciences, College of Dentistry, Jazan University Jazan, Kingdom of Saudi Arabia

${ }^{4}$ Department of Oral Pathology and Microbiology, Sri Venkateswara Dental College and Hospital, Chennai, Tamil Nadu, India

Corresponding Author: A Thirumal Raj, Department of Oral Pathology and Microbiology, Sri Venkateswara Dental College and Hospital, Chennai, Tamil Nadu, India, e-mail: thirumalraj666@gmail.com
In addition to regulating cellular proliferation, Erk1/2 activation leads to increase in urokinase plasminogen activator (uPA) expression which leads to disruption of basement membrane and destruction of the extracellular matrix. ${ }^{2}$ Being a protease, uPA can convert plasminogen to plasmin, thereby regulating cell migration through the modulation of cell membrane adherence with the surrounding extracellular matrix. It also plays a role in normal tissue repair, cancer invasiveness, and metastasis. ${ }^{2}$ A correlation between an increased expression of uPA and its receptors with tumor invasiveness and tumor stage has been reported. ${ }^{2}$ Nakamura et al showed that in LLC-PK1 porcine renal cells, CT-induced Erk1/2 phosphorylation by activating the protein kinase $\mathrm{C}$ (PKC) pathway and thereby resulting in higher expression of uPA (Flow Chart 1). On the contrary, in MDA-MB-231 human breast cell lines, CT inactivated c-Raf via protein kinase A (PKA) pathway and interfered with Erk1/2 phosphorylation, thereby resulting in reduced uPA expression (Flow Chart 1). ${ }^{3}$ The MDA-MB231 human breast cell lines, which are not sensitive to $C T$, exhibit increased invasiveness and represent advancedstage breast cancer. Thus, CT has dual functions, namely cell type-specific up- and downregulation of Erk signaling and urokinase-type plasminogen activator expression depending on the effects on gene expression. ${ }^{3}$

Calcitonin may induce malignancy in cells lacking phosphorylated Erk1/2 signaling. Han et $\mathrm{al}^{2}$ showed a direct effect of $\mathrm{CT}$ on the invasiveness of breast cancer cells and observed that CT-treated breast cancer cells

Flow Chart 1: Calcitonin-mediated tumor inhibition and progression

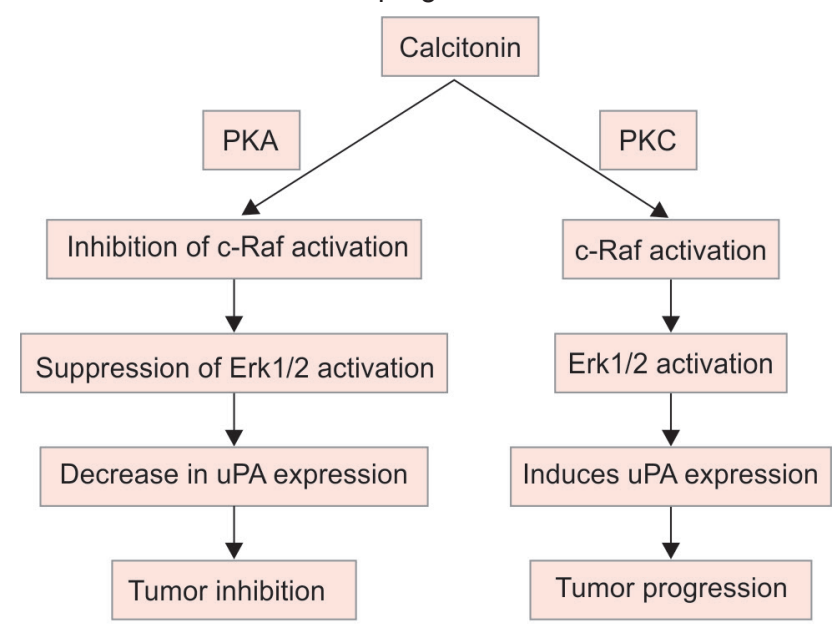

PKA-protein kinase A, pKC-protein kinase C,

Erk1/2- Extracellular signal regulated kinases 1 and 2, uPA-urokinase plasminogen activator 
T47D showed inhibition of cell proliferation. The CT treatment caused a downregulation of UPA and urokinase plasminogen activator receptor in MDA-MB-231 cells, thus inhibiting its invasiveness in vitro. The authors also observed that the treatment of MDA-MB-231 xenograft cells with CT resulted in a reduced uPA mRNA expression in vivo. They suggested that inhibition of tumor invasion was concentration-dependent, seen in only those cell cultures treated with CT for a longer duration (nearly a day). ${ }^{3}$ It is believed that CT alters the phenotype of MDAMB-231 cells, thereby decreasing its invasive potential. ${ }^{3}$ Advanced cases with tumor invasion through lymphatics and metastasis to lymph nodes showed a reduced CTR mRNA expression. ${ }^{2}$ Segawa et al. ${ }^{4}$ reported that in DU145 prostate cancer cells, CT inhibited the phosphorylation of protein kinase. It is said that the MAP kinases cascade inhibition mediated by the PKA pathway was inhibited by the CT treatment and activation of the CTR. Earlyresponse genes, c-fos, and c-jun may be controlled by CT which is mediated by MAPK cascade. ${ }^{4} \mathrm{G}$ proteins compete for the CTR binding sites and this ability affects signal transduction by CT, depending on the different types of cells. The growth of breast cancer cell line T47D is halted by CT. In addition, CT inhibits the epidermal growth factor or insulin which stimulates the tumor growth in these cell lines. Tissue inhibitors of metalloproteases and parathyroid hormone-related protein which regulate the skeletal metastasis are also regulated by CT in breast cancer. ${ }^{3}$ Thus, to conclude, in CTR-expressing cancers, CT could be a potential therapeutic option to control tumor progression.

\section{REFERENCES}

1. Chigurupati S, Kulkarni T, Thomas S, Shah G. Calcitonin stimulates multiple stages of angiogenesis by directly acting on endothelial cells. Cancer Res 2005 Sep; 65(18):8519-8529.

2. Han B, Nakaura M, Zhou G, Ishii A, Nakammura A, Bai $Y$, Mori I, Kakudo K. Calcitonin inhibits invasion of breast cancer cells: involvement of urinokinase-type plasminogen activator (uPA) and uPA receptor. Int J Oncol 2006 Apr;28(4): 807-814.

3. Nakamura M, Utsunomiya H, Nomura S, Kono R, Kakudo K. The two functions of calcitonin: cell type-specific up-and down-regulation of ERK signalling and urokinase-type plasminogen activator expression. J Basic Clin Med 2015;4(1): 25-31.

4. Segawa N, Nakamura M, Nakamura Y, Mori I, Katsuoka Y, Kakudo, K. Phosphorylation of mitogen-activated protein kinase is inhibited by calcitonin in DU145 prostate cancer cells. Cancer Res 2001 Aug;61(16):6060-6063. 